\title{
High-throughput characterization of chemical-associated embryonic behavioral changes predicts teratogenic outcomes
}

\author{
David M. Reif ${ }^{1}(1) \cdot$ Lisa Truong $^{2} \cdot$ David Mandrell $^{2} \cdot$ Skylar Marvel $^{1}$ • \\ Guozhu Zhang ${ }^{1} \cdot$ Robert L. Tanguay ${ }^{2}$
}

Received: 4 May 2015 / Accepted: 9 June 2015 / Published online: 1 July 2015

(C) The Author(s) 2015. This article is published with open access at Springerlink.com

\begin{abstract}
New strategies are needed to address the data gap between the bioactivity of chemicals in the environment versus existing hazard information. We address whether a high-throughput screening (HTS) system using a vertebrate organism (embryonic zebrafish) can characterize chemical-elicited behavioral responses at an early, 24 hours post-fertilization (hpf) stage that predict teratogenic consequences at a later developmental stage. The system was used to generate full concentration-response behavioral profiles at $24 \mathrm{hpf}$ across 1060 ToxCast $^{\mathrm{TM}}$ chemicals. Detailed, morphological evaluation of all individuals was performed as experimental follow-up at 5
\end{abstract}

Electronic supplementary material The online version of this article (doi:10.1007/s00204-015-1554-1) contains supplementary material, which is available to authorized users.

Robert L. Tanguay

robert.tanguay@oregonstate.edu

David M. Reif

dmreif@ncsu.edu

Lisa Truong

lisa.truong@oregonstate.edu

David Mandrell

mandrelld@gmail.com

Skylar Marvel

swmarvel@ncsu.edu

Guozhu Zhang

gzhang6@ncsu.edu

1 Bioinformatics Research Center, Department of Biological Sciences, North Carolina State University, Raleigh, NC, USA

2 Sinnhuber Aquatic Research Laboratory, Department of Environmental and Molecular Toxicology, Environmental Health Sciences Center, Oregon State University, 28645 East Highway 34, Corvallis, OR 97333, USA days post-fertilization (dpf). Chemicals eliciting behavioral responses were also mapped against external HTS in vitro results to identify specific molecular targets and neurosignalling pathways. We found that, as an integrative measure of normal development, significant alterations in movement highlighted active chemicals representing several modes of action. These early behavioral responses were predictive for 17 specific developmental abnormalities and mortality measured at $5 \mathrm{dpf}$, often at lower (i.e., more potent) concentrations than those at which morphological effects were observed. Therefore, this system can provide rapid characterization of chemical-elicited behavioral responses at an early developmental stage that are predictive of observable adverse effects later in life.

Keywords Developmental neurotoxicology $\cdot$ Alternative testing $\cdot$ Chemical biology $\cdot$ Behavior $\cdot$ Zebrafish $\cdot$ Highthroughput screening · Bioinformatics · Data integration . ToxCast $\cdot$ Bioactivity

\section{Introduction}

There are tens of thousands of manufactured chemicals currently in commerce, each with varying production volumes, use profiles, and potential for environmental release, persistence, and bioaccumulation. Comprehensive data on the human and environmental health hazards of these chemicals remain elusive, ranging from sparse to nonexistent. High-throughput screening (HTS) efforts such as ToxCast ${ }^{\mathrm{TM}}$ and Tox $21^{\mathrm{TM}}$ were conceived to begin addressing this data need by applying new in vitro assay technologies to speed the pace of chemical testing (Collins et al. 2008; Judson et al. 2010). 
While simple in vitro screening assays are useful for probing targeted receptor-ligand interactions, these screens fail to take into account the complexity of the vertebrate nervous system and will miss chemicals that modify nervous system function in novel ways. A phenotype-based screen is needed to identify developmental neuromodulatory compounds in the absence of specific targets (Burns et al. 2013; Selderslaghs et al. 2013). Designing in vivo phenotypic screens in a high-throughput manner would provide the ability to discover complex behavioral phenotypes using whole organisms, where the full gamut of coordinated events leading to nervous system development occurs, including cellular differentiation, proliferation, migration, synapse formation, and apoptosis (Makris et al. 2009; Padilla et al. 2012; Sanes et al. 2005; Truong et al. 2014).

Zebrafish (Danio rerio) is a prolific, small, complex organism that shares a highly conserved anatomy and physiology with all vertebrates (Howe et al. 2013). Importantly, the critical processes of zebrafish neurodevelopment are homologous to those in humans (Tropepe and Sive 2003). Early in zebrafish embryogenesis [roughly 19-29 hours post-fertilization (hpf)], spontaneous tail contractions occur as the muscles in this region are innervated (Kimmel et al. 1995). This spontaneous behavior is sensitive to high-intensity light perturbation via photoreceptors in the developing hindbrain and has been designated as the photomotor response (PMR) (Kokel et al. 2013). The normal PMR is sensitive to chemical perturbation and amenable to screening for behavior-modifying compounds (Raftery et al. 2014). In Kokel et al. (2010), the authors developed behavioral barcodes that classified small molecules from six libraries (primarily pharmaceuticals and candidate pharma-like chemical entities) into related clusters according to PMR of pooled embryo samples at a single concentration.

Here, we report HTS results that characterize the diverse ToxCast Phase-I and Phase-II libraries (1060 unique chemicals) using a five-point, log concentration range (from $6.4 \mathrm{nM}$ to $64 \mu \mathrm{M}$ ) for each chemical. These results were generated using single-embryo wells with 32 replicate samples at each concentration, where all individuals are followed up by morphological assessments at $120 \mathrm{hpf}$ (5 dpf). Thus, our assay system allows the characterization of behavioral changes across chemical concentrations, provides increased detection power and reliability of individual endpoint measurements, and quantifies developmental consequences of behavioral aberrations measured early in development. As additional follow-up, we mapped our results to external data to discover associations between behavioral responses and in vitro endpoints targeting specific biological pathways.

\section{Methods}

\section{Chemicals}

The ToxCast Phase-I and Phase-II chemical libraries were provided by the US EPA National Center for Computational Toxicology (NCCT). There were 1060 unique chemicals from various sources. Stock solutions of all the chemicals were provided at a $20-\mathrm{mM}$ concentration in $100 \%$ dimethyl sulfoxide (DMSO) in multiple 96-well plates. More details regarding the chemical library and quality control can be found at http://www.epa.gov/NCCT/toxcast/chemicals.html. Chemical dilutions (1:10) for exposure plates were made using DMSO and standard embryo medium (EM) (Westerfield 2000) to achieve a concentration range of $0.064-640 \mu \mathrm{M}$ with a DMSO concentration of $6.4 \%$. All dilution plates were sealed and then stored at $-20{ }^{\circ} \mathrm{C}$ until used for exposure.

\section{Experimental design}

Adult wild-type zebrafish (Tropical 5D) were raised in the Sinnhuber Aquatic Research Laboratory at Oregon State University, Corvallis, Oregon. Groups of 1000 adult zebrafish were housed in 100 gallon tanks kept at standard laboratory conditions of $28{ }^{\circ} \mathrm{C}$ on a 14-h light/10-h dark photoperiod in fish water (reverse osmosis water supplemented with Instant Ocean ${ }^{\mathrm{TM}}$, a commercially available salt). Zebrafish were spawned, and embryos were collected and staged according to Kimmel et al. (Kimmel et al. 1995). At $4 \mathrm{hpf}$, embryos were dechorionated using pronase $(63.6 \mathrm{mg} / \mathrm{ml},>3.5 \mathrm{U} / \mathrm{mg}$, Sigma-Aldrich: P5147) by a custom automated dechorionator (Mandrell et al. 2012).

Using the automated embryo placement systems (AEPS) previously described (Mandrell et al. 2012), $6 \mathrm{hpf}$ dechorionated embryos were individually placed into a 96-well plate ( 1 embryo per well) prefilled with $90 \mu \mathrm{L}$ of EM. Ten microliters of chemical from dilution plate 2 was added to each row of two 96-well plates. A final DMSO concentration was maintained throughout the experiment of $0.64 \%$ $(v / v)$. For each concentration, a total of 32 embryos were exposed. All exposure plates were sealed using parafilm to prevent evaporation and wrapped in aluminum foil to prevent light exposure. During this development period, zebrafish embryos are able to adapt to the dark and develop normally (Kokel et al. 2013). Exposed plates were stored in $28{ }^{\circ} \mathrm{C}$ incubator, and embryos were statically exposed until $120 \mathrm{hpf}$ (5 dpf). At $24 \mathrm{hpf}$, embryos were assessed for PMR (as described below) and evaluated for mortality $(\mathrm{MO} 24=$ mortality at $24 \mathrm{hpf})$ (Truong et al. 2011). At $5 \mathrm{dpf}$, embryos were assessed for 17 morphological endpoints and collected in Zebrafish Acquisition and Analysis Program (ZAAP) (Truong et al. 2014). 
Embryos were assessed for PMR using the custom-built Photomotor Response Analysis Tool (PRAT). The system uses a Prosilica GX3300 (Allied Vision, Stadtroda, Germany) with near infrared (NIR) band-pass filter to remove any influence of stimulus light. The lens is double telecentric (Navitar, Rochester, New York), mounted in an inverted manner beneath the plate holder to allow for imaging with minimum distortion and perspective interference. Imaging illumination is accomplished with a NIR (850 nm) Backlight (Smart Vision Lights, Muskegon, MI). PMR stimulus light is from two white L300 Linear Lights (Smart Vision Lights, Muskegon, MI). The system is controlled by custom hardware for timing the high-intensity light stimuli and system backlight. Video recording began immediately prior to light cycle initiation and captures 850 frames of digital video, recorded at 17 frames s-1. The light cycle consists of $30 \mathrm{~s}$ ( $\mathrm{sec}$ ) of background (prior to the first light pulse), a short pulse of light, $9 \mathrm{~s}$ before the next pulse, a second pulse of light, and then $10 \mathrm{~s}$ of dark.

To analyze the videos, a custom MATLAB program (Mathworks, Natick, MA) was used to compute a movement index for each frame stamp (the pixel differences between frames). The program output was processed using custom R scripts ( $\mathrm{R}$ Core Team 2014) to discard the lag time that occurs between when the video recording was initiated and the light cycle begins/ends. A time stamp was created from the frame stamp by taking the first stamp after the recording started, setting that as $0 \mathrm{~s}$, and then taking the floor of the sequential frame stamps.

\section{Quality control, identifying outliers, and quantifying expected responses}

All subsequent analysis began with the processed data described above and was implemented using the $\mathrm{R}$ language (R Core Team 2014). First, we checked for aberrant background responses in individual plates and chemical sets by comparison with global patterns across all experiments. A total of 66 chemical sets (approximately $6 \%$ ) were rerun in full (both plates) after evaluation of the controls to identify those lacking post-light excitatory responses via either background (pre-light) peak movement index higher than the excitatory (post-light) peak, or no difference in movement from background to excitatory interval. After confirming lack of batch effects, these rerun data overwrote the original data in the results reported here.

Next, we removed wells annotated as having embryo mortality (MO24), as described in Experimental Design. For the analysis, the recorded periods were truncated to assure equivalence in recorded experimental period for all chemicals. Outlier detection was applied independently to each chemical concentration and time point combination. Movement values between the top 80-99 \% quantiles (ignoring zero-movement values) were fit by a shifted gamma distribution using a maximum likelihood approach. An outlier was then defined as any movement value with a Bonferroni-corrected $p$ value $<0.05 / n$, where $n$ is the number of movement values used to fit the model. This stringent cutoff criterion was chosen to remove outliers while preserving natural variation.

Additionally, we implemented a filter based upon departure from expected responses across all negative control (i.e., DMSO-only) wells. This filter prevents any significant calls from being made when the control fish are less responsive than expected. The peak-mean movement values within the excitatory interval were fit to a gamma distribution using a maximum likelihood approach, where a peak-mean movement value was defined as the maximum mean movement value of exposed fish for each chemical within that interval. Significance calls were prevented for any chemical with a peak-mean movement $p$ value $<0.05$.

\section{Analysis of chemical-associated activity}

Our analysis was designed to account for the unusual distributional properties of developmentally normal, spontaneous movement responses, and avoid bias toward one side of a bidirectional behavioral response where either direction could represent aberrant health effects of a chemical. Postfiltering (described in the previous section), the remaining experimental period (21-48 s) was subdivided according to light pulses: background $(\mathrm{B})=9 \mathrm{~s}$ prior to first pulse (21-29 s); pulse $_{1}=$ first pulse of light; latency ${ }_{1}=1 \mathrm{~s}$ immediately following first pulse; excitatory $(\mathrm{E})=8 \mathrm{~s}$ between light pulse $(32-39 \mathrm{~s})$; pulse $_{2}=$ second light pulse; latency $_{2}=1 \mathrm{~s}$ immediately following second pulse; refractory $(\mathrm{R})=7 \mathrm{~s}$ after second pulse $(42-48 \mathrm{~s})$.

The statistical analysis of activity considered only the background $(\mathrm{B})$, excitatory $(\mathrm{E})$, and refractory $(\mathrm{R})$ intervals. The overall pattern of activity within each $\mathrm{B}, \mathrm{E}$, or $\mathrm{R}$ interval was compared to that interval's negative control ( $0 \mu \mathrm{M})$ activity using a combination of percent change ( $-50 \%$ change from control for hypoactivity; $75 \%$ change from control for hyperactivity) and a Kolmogorov-Smirnov test (Bonferroni-corrected $p$ value threshold $=0.05 / 5$ concentrations $=0.01)$. The percent change thresholds for hypo- and hyperactivity were parameterized so that the distributions of negative control responses were equivalent across activity-associated chemicals (i.e., "hits"). The Kolmogorov-Smirnov test compared the empirical cumulative distribution function (eCDF) between the chemical-treated samples and the negative control. For each chemical concentration set of $n=32$ embryo wells, the treated wells were compared to vehicle (negative control) wells across two replicate plates for each chemical. This permits scalability of the procedure to custom batches of chemicals 
in either new experiments or retests (see "Quality control, identifying outliers and quantifying expected responses").

The $5 \mathrm{dpf}$ developmental morphology data, as described in Truong et al. (2014), were used to estimate the predictive value of this early, $24 \mathrm{hpf}$ response. We modified the calculation of statistical significance from that paper in order to account for changing proportions from censored wells. Fisher's exact test was used to define a morphology lowest effect level (LEL) if the incidence of the $5 \mathrm{dpf}$ morphological endpoints were significantly different between the control fish and a concentration of chemical. Fish indicated as MO24 or $5 \mathrm{dpf}$ mortality (MORT) were not included in the count data used in the test. The relative risk (RR) associating movement at $24 \mathrm{hpf}$ (early effects) and the morphological endpoints measured at $5 \mathrm{dpf}$ (later effects) was estimated from the number of chemicals positive for both early and late effects (true positive $=\mathrm{TP}$ ); positive for early effects but negative for later effects (false positive $=$ FP); negative for early effects but positive for later effects (false negative $=\mathrm{FN}$ ); and negative for both early and late effects (true negative $=\mathrm{TN}$ ), as:

$$
\left(\frac{\mathrm{TP}}{\mathrm{TP}+\mathrm{FP}}\right) /\left(\frac{\mathrm{FN}}{\mathrm{FN}+\mathrm{TN}}\right) \text {. }
$$

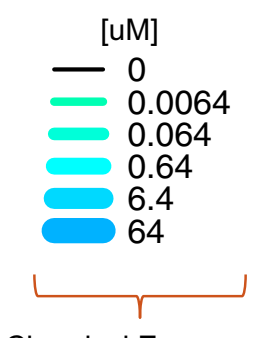

Chemical Exposure

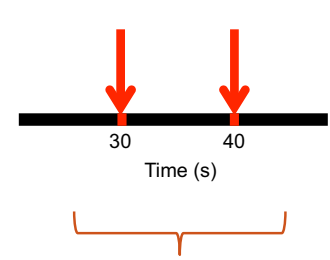

Light Pulse Exposure

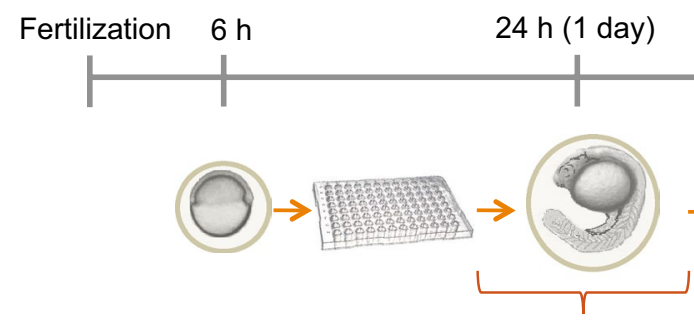

Behavioral Assessment

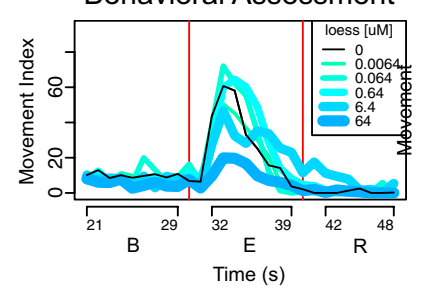

Fig. 1 Experimental design. For each of 1060 chemicals, the chemical exposure at $6 \mathrm{hpf}$ saw one of 5 concentrations of a given chemical added to individual embryo wells in 96-well plates. At $24 \mathrm{hpf}$, plates were subjected to light pulse exposure (at $30 \mathrm{~s}$ and $40 \mathrm{~s}$ ) for the PMR assay. Movement was recorded across the short $(<1 \mathrm{~min})$ interval and

\section{Results}

Data were collected from embryos exposed to each of 1060 ToxCast Phase-I and Phase-II chemicals (see "Chemicals"). The experimental design in Fig. 1 illustrates dechorionated embryonic zebrafish exposed to vehicle control plus five concentrations at $6 \mathrm{hpf}$, a photomotor response (PMR) assay at $24 \mathrm{hpf}$, and evaluation of 17 developmental malformation endpoints at $5 \mathrm{dpf}$. The response to the $24 \mathrm{hpf}$ behavioral assay was interrogated, and movement profiles were established that characterized bidirectional responses to chemical exposure across a common five-point concentration range (see "Methods"). Representative hypo-/hyperactivity-associated chemical profiles are shown in Fig. 2. Full concentration response results for all chemicals are given in Supplemental Table 1.

\section{Global patterns of response and reliability}

While individual sample (embryo) wells displayed variability in the magnitude of response, by considering the nonparametric distribution of all samples, a remarkably consistent pattern of activity emerged in control wells (see 
(A) Hyperactivity example
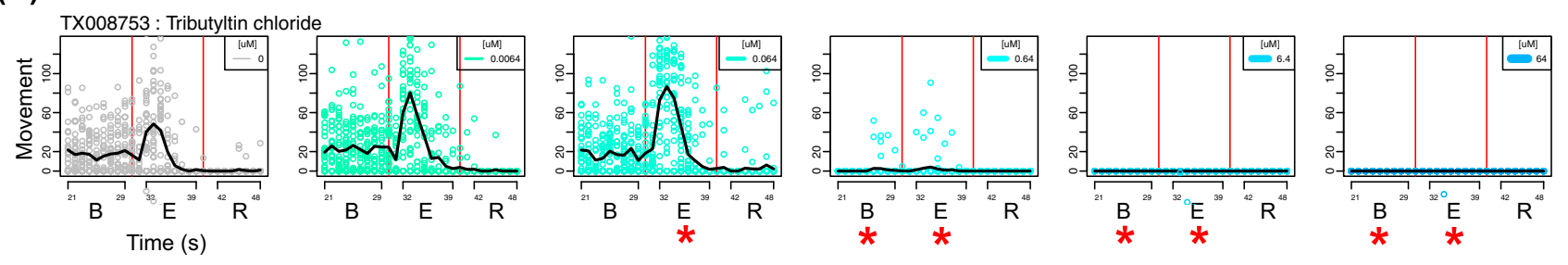

(B) Hypoactivity example
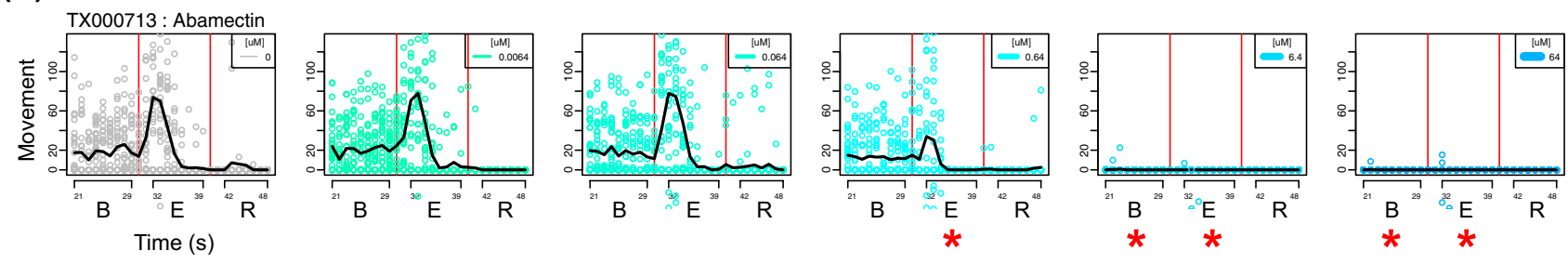

Fig. 2 Detecting significant alterations in movement. The top panel shows movement (vertical axis, dimensionless index) versus time (horizontal axis, in sec) across all concentrations, increasing from left (control wells in the absence of chemical) to right (highest concentration $=64 \mu \mathrm{M})$. The vertical red lines indicate the timing of light pulses. Each circle represents movement at a given time for a replicate embryo well (32 replicates per chemical concentration combination). A locally weighted regression (LOESS) fit was plotted as a solid black line. The experimental intervals modeled in the behavioral

leftmost plots in Fig. 2). The background (B) interval was characterized by basal activity with moderate variance but absence of distinct activity peaks. The excitatory (E) interval displayed a single peak of hyperactivity with high variability as to the absolute peak movement and then immediately dropped to a movement level below that of $\mathrm{B}$. The refractory $(\mathrm{R})$ interval was characterized by a significant drop in movement compared to $\mathrm{B}$, with inactivity lasting beyond the time interval measured.

The 9 sets of embedded chemical triplicate (each sourced separately) included in the data were used to assess reliability of activity calls. Using the same metric of assay concordance estimated for the ToxCast ${ }^{\mathrm{TM}}$ in vitro systems (http://epa.gov/ncct/toxcast/data.html), the reliability of activity calls was $89 \%$. For example, all three replicate sets for Allethrin exhibited the extremely rare refractory (R) hyperactivity response (observed in less than $1 \%$ of all chemicals) at the same $64-\mu \mathrm{M}$ concentration. Therefore, we concluded that the reliability was sufficient to collapse the embedded triplicate and report subsequent results with respect to the set of 1,060 unique substances.

In total, 296 chemicals showed statistically significant activity in at least one interval (Supplemental Table 1 and Fig. 3). Of these significant chemicals, considering the lowest effect level (LEL) as the most potent (i.e., smallest concentration of chemical) response within a given time interval across all concentrations, there were 148 hypoactive

analysis included background (B), excitatory (E), and refractory (R). Red asterisks denote significant responses for a particular concentration-time interval combination. The hyperactivity example chemical shown, tributyltin chloride, had an estimated lowest effect levels (LEL) of $0.064 \mu \mathrm{M}$ for hyperactivity within the E interval and an LEL of $0.64 \mu \mathrm{M}$ for hypoactivity within the B interval. The hypoactivity example chemical shown, abamectin, had estimated LELs of $6.4 \mu \mathrm{M}$ in $\mathrm{B}$ and $0.64 \mu \mathrm{M}$ in $\mathrm{E}$ (color figure online)

and 5 hyperactive in $\mathrm{B}, 260$ hypoactive and 18 hyperactive in $\mathrm{E}$, and 7 hyperactive in R. The LEL overlap across intervals consisted of 12 out of 153 that were unique to $\mathrm{B}, 136$ of 278 that were unique to $\mathrm{E}$, and 6 of 7 that were unique to R. Beyond totals, considering the LEL as the most potent response across time intervals for a given chemical revealed the $\mathrm{E}$ interval as most sensitive in terms of detecting the most chemicals (171) at the lowest concentration. The B interval was the most sensitive for only 13 chemicals, and although rare, the 7 chemicals hyperactive within the $\mathrm{R}$ interval defined the LEL across intervals. These results indicate that each experimental period is differentially informative and that light-elicited behavioral changes in $\mathrm{E}$ tend to be most sensitive to chemical perturbation.

The LEL was chosen as the unit of analysis within each interval for several reasons illustrated by Fig. 3. The most sensitive effect (i.e., defining an LEL as the most potent chemical concentration associated with a significant effect) should be the most specific behavioral indicator. While hypoactive behavioral effects were observed for some chemicals at higher concentrations than those at which a hyperactive LEL was observed, zero chemicals across this diverse 1060 set caused significant reduction in movement followed by hyperactivity at a higher concentration. This point is extremely important when considering a behavioral endpoint that can take on three states (i.e., hyperactive, hypoactive, or insignificant change), because the 

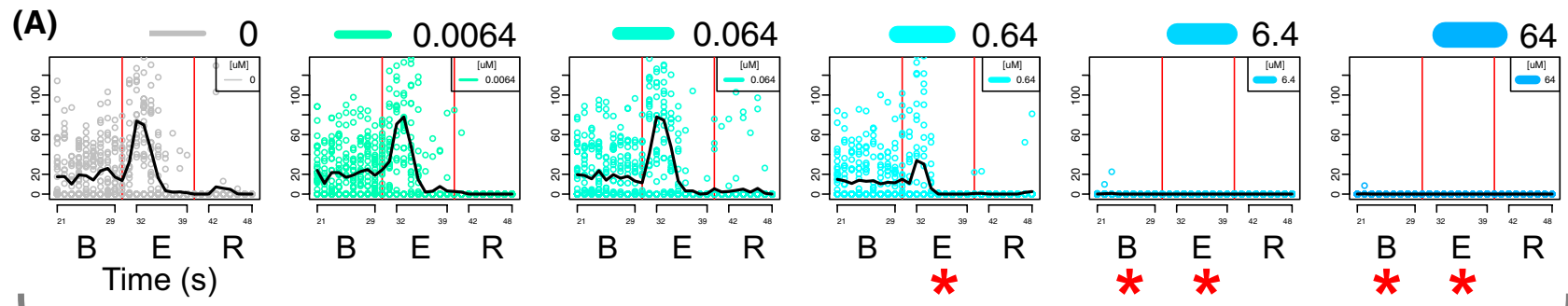

(B)

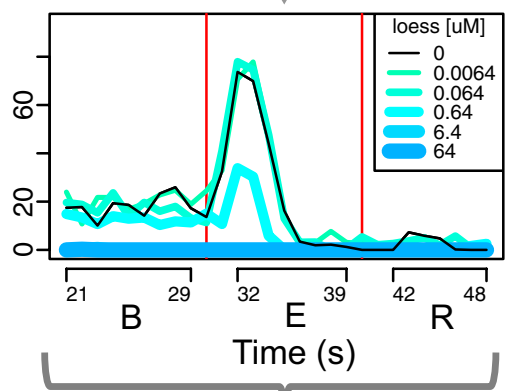

(C)

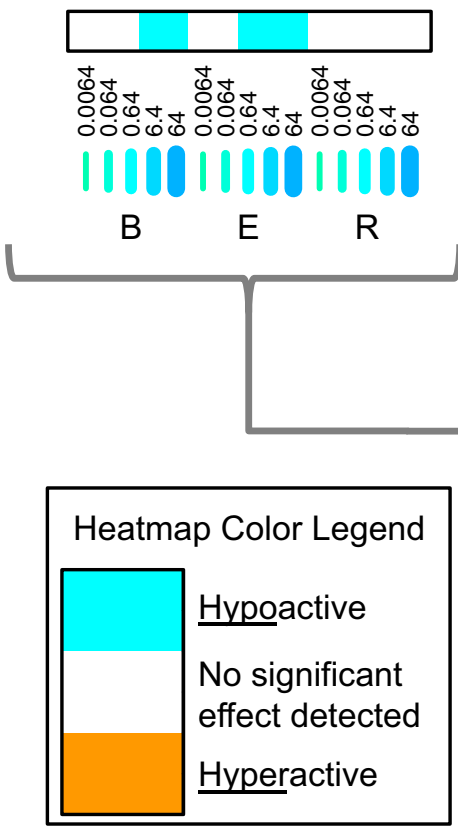

(D)

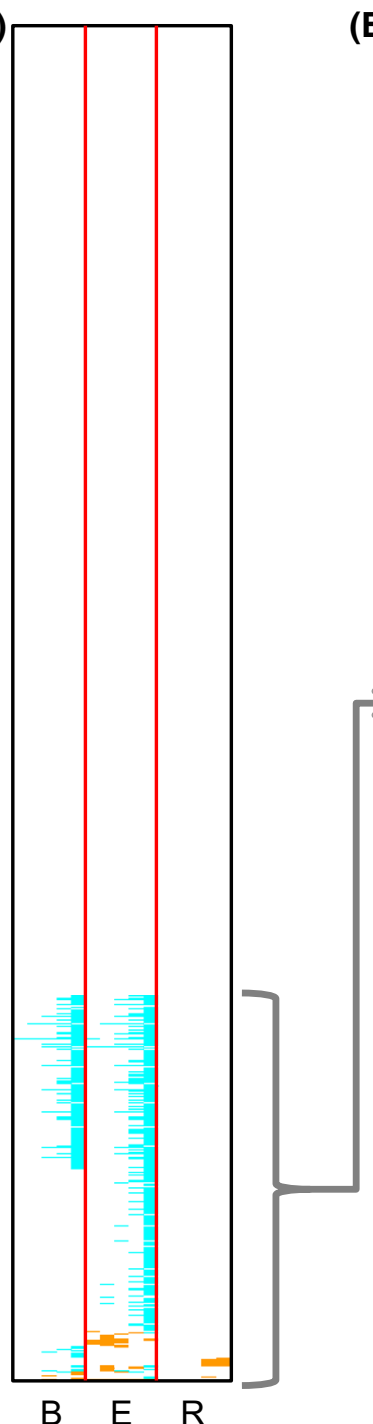

(E)

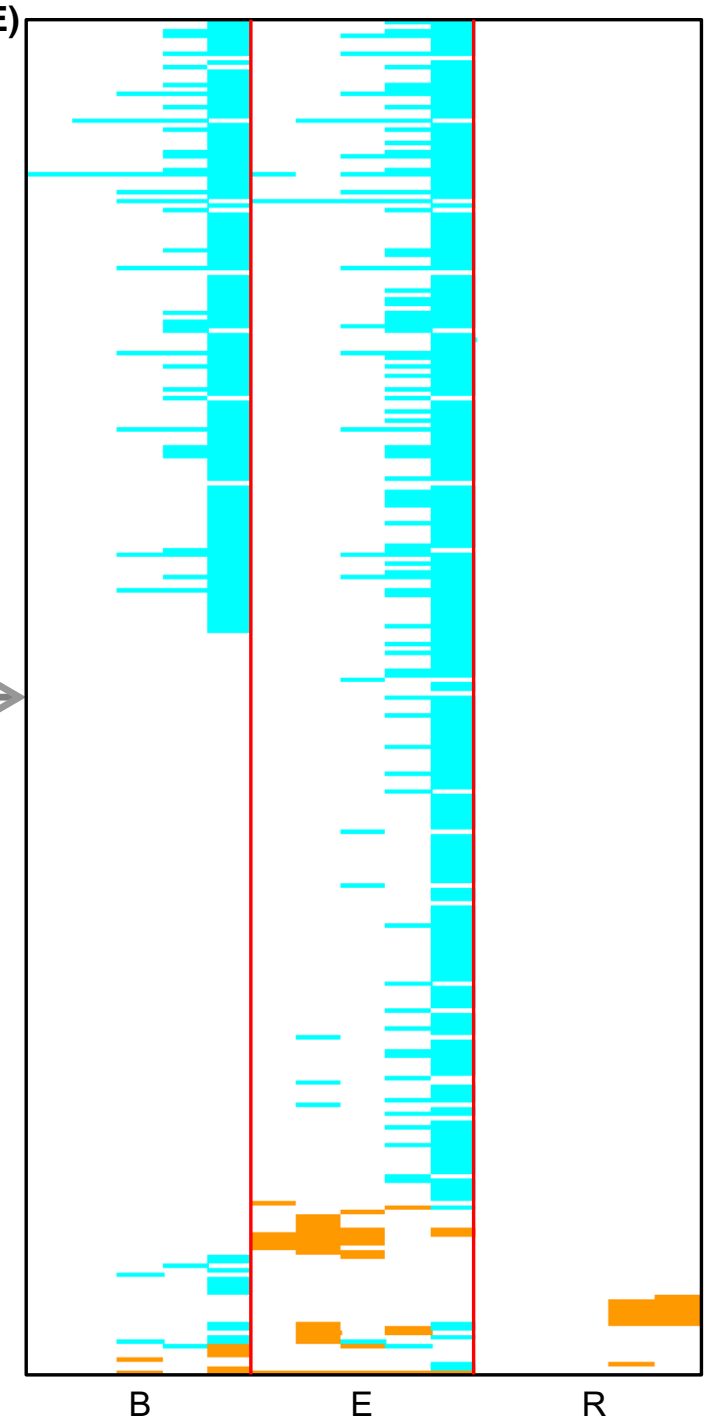

Fig. 3 Response patterns for all chemicals across concentrations. In part A, movement versus time is plotted for all concentrations from left (control wells in the absence of chemical) to right (highest concentration) for a single chemical (abamectin, as in Fig. 2). Red asterisks denote significant responses for a particular concentration-time interval combination. In part $\mathrm{B}$, the concentration-response data for the given chemical have been overlaid as a locally weighted regression (LOESS) fit, plotted with line thickness proportional to concentration of chemical. The LOESS fit for control embryos (wells in the absence of chemical) is plotted as a solid black line. In part $\mathrm{C}$, the significant responses are collapsed into a heatmap vector (row) colored according to hyperactivity (orange) or hypoactivity (blue) at a given concentration and time interval. For the given chemical, only hypoactive significant responses were observed. In part $\mathrm{D}$, the significant response vector for the given chemical is added as a single row in a heatmap for all chemicals (one chemical per row). The colorless portion of the heatmap represents the 764 chemicals without significant activity. In part E, the portion of the heatmap containing the 296 chemicals showing significant activity within at least one interval has been expanded (color figure online) 
dose-response may appear "non-monotonic" with respect to absolute movement. For example, a low-dose hyperactive response may trend toward hypoactivity at higher doses (e.g., tributyltin chloride or butylparaben), but the intermediate doses may be observed to pass through an apparent "no effect" response. This finding highlights the importance of a concentration-response design in assessing behavior, where a single concentration may happen to coincide with the point at which a response is neither significantly hyper- nor hypoactive as it transitions from significant hyperactivity at lower concentrations on the way to hypoactivity at higher concentrations. Moreover, because many of these high-concentration movement reductions were associated with mortality, it is likely that these effects were indicative of toxicity pathways leading to death.

Considering hypo- or hyperactivity LELs defined over the light intervals specified, we classified chemicals as eliciting light-independent movement alterations, lightdependent movement alterations (i.e., PMR specific), or indistinguishable from the observed data. The concentration-response profiles allowed us to infer light dependence for chemicals eliciting a post-light response (in E or R) at a concentration more potent than that at which significant (or absence of) B responses were observed. We found that 178 chemicals met this criterion of light-dependent movement alteration as the most sensitive effect (Supplemental Table 2). The LELs were distributed as 25 hyperactive and 153 hypoactive. The chemicals included several conazoles, thiocarbamates, metals, phthalates, toluenes, and the androgenic steroids 17-methyltestosterone, 17beta-trenbolone, 4-androstene-317-dione, and 5alpha-dihydrotestosterone.

In addition to evaluating signature profiles, we assessed the association of a chemical's tendency to partition between hydrophobic and hydrophilic phases (LogP) with its ability to impact nervous system development in the PMR assay. Chemicals with higher LogP should be more permeable across biological membranes. However, as with a recent meta-analysis of zebrafish results across laboratories and experimental designs (Ducharme et al. 2013), we did not find significant evidence of $\operatorname{LogP}$ trending with effects across all chemicals in this behavioral assay. The absence of observed correlation may be due in part to the dechorionation step prior to chemical exposure, which facilitates uptake.

\section{Diverse modes of action are detectable by this assay}

Examining chemicals having known bioactivity that displayed particular response patterns across intervals suggests that several modes of action are detectable by this assay, including direct binding to neurological elements, indirect signaling disruption, muscular contractile disregulation, and disruption of normal structural development.
For example, the PMR assay was adept at detecting insecticides shown to target acetylcholinesterase, such as malathion (Cook et al. 2005) and all three forms of chlorpyrifos tested (Levin et al. 2003; Li et al. 2014; Yang et al. 2011). Moreover, this assay corrected identified the oxon metabolite of chlorpyrifos as more potent than the parent compound (6.4 vs. $64 \mu \mathrm{M}$, respectively). The thiocarbamate pesticides cycloate, dazomet, disulfiram, molinate, sodium dimethyldithiocarbamate, thiobencarb, thiram, and vernolate, known to cause notochord malformation resulting in neuronal and muscular disruptions, were identified by this assay but not associated with mortality (Haendel et al. 2004; Tilton et al. 2006; Tilton and Tanguay 2008). Not only does the PMR assay detect strictly nervous system or canonical neurotoxic modes of action, but it also identifies chemicals disrupting gross structural development, such as tributyltin chloride, tributyltin methacrylate, and triphenyltin hydroxide, which showed behavioral effects at concentrations lower than those at which morphological abnormalities were observed (McGinnis and Crivello 2011; Micael et al. 2007).

We also find several examples of chemical structural classes related to activity. None of the eight compounds from the thiophosphate alkyl subclass (tribufos, chlorethoxyfos, disulfoton, ethion, phorate, fosthiazate, terbufos, ethoprop) were active at $24 \mathrm{hpf}$. However, eighteen other thiophosphates, including fenitrothion, fenthion, methyl parathion, parathion (metabolized in vivo to Paraoxon), azinphos-methyl (gusathion), malathion, and methidathion, were all hypoactive in the post-light E interval, presumably via known acetylcholinesterase-related mechanisms. All five strobins included in this chemical set showed evidence of hypoactivity, with fluoxastrobin, trifloxystrobin, pyraclostrobin, and picoxystrobin having significant effects in the B and E intervals, and azoxystrobin's otherwise significant hypoactivity censored by excess MO24 (mortality at $24 \mathrm{hpf}$ ). This may represent a mitochondrial adverse outcome pathway (AOP), where these fungicides act to inhibit the respiratory chain at the level of complex III (Bartlett et al. 2002). Indeed, beyond these strobins, other chemicals with known mitochondrial targets, such as rotenone (Ai et al. 2014; Navarro et al. 2010), pyridaben (Navarro et al. 2010), and fenpyroximate (Z,E) (Shiraishi et al. 2012) displayed hypoactive responses.

We found that significant movement within the refractory interval was very rare, with only seven chemicals showing hyperactivity: hexaconazole, carbofuran, cypermethrin, methomyl, prallethrin, S-bioallethrin, and allethrin. The response patterns suggested delayed recovery from light-stimulated excitation or sustained hyperactivity with disruption of the stereotypical photomotor response. These chemicals include 4 pyrethroids known to affect ion channels, a known neurotoxic triazole organophosphate 
(hexaconazole), and two carbamates (methomyl and carbofuran, which was once prescribed as an anxiety treatment in humans) known to affect acetylcholinesterase activity (Ensibi et al. 2014).

The most potent hypoactive chemicals at $24 \mathrm{hpf}$ (LEL $\leq 0.064 \mu \mathrm{L}$ ) were emamectin benzoate (Carmichael et al. 2013), rotenone (Swarnkar et al. 2011), triphenyltin hydroxide (Zsombok et al. 1997), thiram (Lee and Peters 1976), mercuric chloride (Yasutake et al. 2010), dapsone (Waldinger et al. 1984), and fluconazole (van Schie et al. 2011). Although all of these compounds have been implicated in nervous system perturbations related to neuromuscular dysfunction, this behavioral assay detected diverse mechanisms of action. For example, rotenone is a pesticide that promotes Parkinson-like behavior upon exposure by inhibiting mitochondrial complex I (Bretaud et al. 2004), while emamectin benzoate interferes with $\gamma$-aminobutyric acid (GABA)-gated ion channels in the nervous system (Carmichael et al. 2013).

\section{External data integration to identify specific molecular targets}

To investigate whether chemicals associated with neurobehavioral phenotypes in our data were associated with biological pathways that manifest in neuroactive responses, we performed a statistical enrichment analysis of our zebrafish $24 \mathrm{hpf}$ behavioral (in vivo) data in conjunction with biochemical assay (in vitro) results from (Sipes et al. 2013), where the ToxCast Phase-I and Phase-II chemicals were screened across 331 cell-free enzymatic and ligand-binding assay targets. The analysis considered statistical enrichment (Fisher's exact test $p<0.05$ and odds ratios $>1$ ) of chemical "hits" in the $24 \mathrm{hpf}$ behavioral results associated with individual in vitro assays (e.g., Novascreen human peripheral-type benzodiazepine receptor-binding assay). We also performed the same enrichment analysis in association with high-level assay groupings from Table 1 in Sipes et al. which aggregated assays into functional groupings such as aminergic G-protein coupled receptor binding (32 assays), ion channel binding (7 assays), or cholinesterase enzyme inhibition (3 assays).

Considering the full concentration-response profile to define light-dependent effects (see Supplemental Table 2 as described earlier), chemicals eliciting light-dependent LELs were associated with hits on assay groups of nuclear receptors, transporters, CYPs, ion channels, and aminergic GPCRs. The individual assays associated with lightdependent LELs were the binding assays probing VMAT2 (vesicular monoamine transporter), PBR (peripheral benzodiazepine receptor or TSPO), DAT (dopamine transporter), NET (norepinephrine transporter), PXR (pregnane$\mathrm{X}$ receptor), PR (progesterone receptor), AR (androgen receptor), and CAR (constitutive androstane receptor), as well as enzymatic inhibition assays for MAOB (monoamine oxidase B) and several CYPs (human CYP19A1, CYP1A1, CYP2B6, CYP1A2, CYP2D6). This behavioral assay identified light-dependent hypoactivity for 15 of the 16 chemicals that were hits in the external, in vitro MAOB assay.

We also considered more general response prototypes of hyper- or hypoactivity within each experimental light interval as chemical subsets for mapping against the targeted, in vitro results. These response prototypes were defined according to LELs within intervals. For example, 123 chemicals displayed the $(\mathrm{B}, \mathrm{E}, \mathrm{R})$ prototype $=(-1$, $-1,0)$, corresponding to hypoactivity LELs within both the background and excitatory intervals. We found that this prototype indicating general hypoactivity was associated $(p<0.05)$ with many of the same assays as the light-dependent chemicals, plus assays for 5-HT (serotonin receptor) and several opioid receptors. Thus, chemicals eliciting abnormal behavioral responses at $24 \mathrm{hpf}$ in zebrafish are enriched for activity within in vitro assays probing genes that have known roles in canonical neurological signaling. Variation in these genes, such as monoamine oxidase A (MAOA), has been associated with a variety of psychiatric traits and sensitivity to chemical therapeutics (Ziermans et al. 2012).

On the flip side, statistically significant in vitro assay associations with negative chemicals, i.e., those with prototype $=(0,0,0)$, had "protective" odds ratios (Fisher's exact test $p<0.05$ and odds ratios $<1)$. In this context, the interpretation of a significant odds ratio $<1$ is that the compounds inactive in this behavioral assay were less likely to "hit" the given targeted assays. The in vitro assay groups fitting this criterion included cholinesterases, nuclear receptors, transporters, CYPs, ion channels, and aminergic GPCRs. Thus, chemicals that were negative in this $24 \mathrm{hpf}$ behavioral assay displayed essentially the inverse relationship with in vitro assays targeting plausible neuroactivity pathways. Importantly, there were no significant, positive odds ratios that associated the $(0,0,0)$ behavioral prototype with any individual assay or assay group from Sipes et al.

\section{Predictive power of $24 \mathrm{hpf}$ behavioral results}

Taking advantage of the multi-scale nature of the phenotypic measurements collected, we integrated across $24 \mathrm{hpf}$ behavior and follow-up $5 \mathrm{dpf}$ development endpoint to understand relationships amongst endpoint types. Figure 4 shows the prospective association relative risk (RR) between hypoactive movement at $24 \mathrm{hpf}$ and the morphological endpoints measured at $5 \mathrm{dpf}$. We found that these early behavioral responses were predictive (RR $p$ value $<0.05)$ of 17 specific developmental 


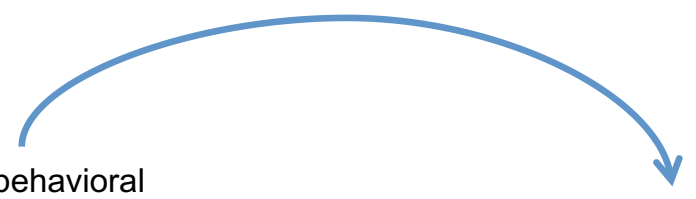

1 day behavioral endpoints

5 day developmental endpoints

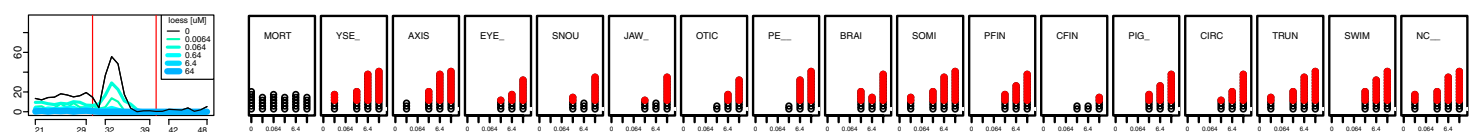

\section{Specific developmental endpoints at $5 \mathrm{dpf}$}

MORT: mortality

YSE: yolk sac edema

AXIS: bent body axis

EYE: eye

SNOU: snout

JAW: jaw

OTIC: otic

PE: pericardial edema

BRAI: brain

SOMI: somite

PFIN: pectoral fin

CFIN: caudal fin

PIG: pigmentation

CIRC: circulation

TRUN: trunk length

SWIM: swim bladder

NC: notochord distortion
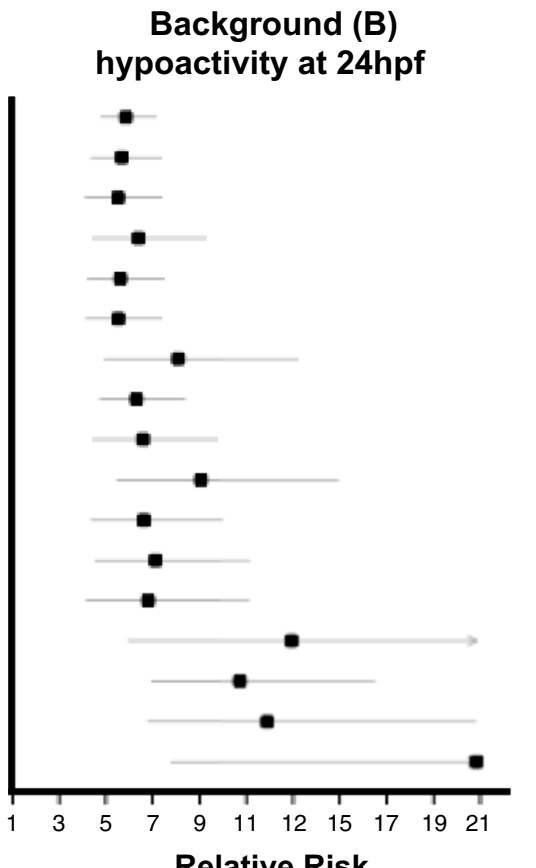

Excitatory (E) hypoactivity at $24 \mathrm{hpf}$

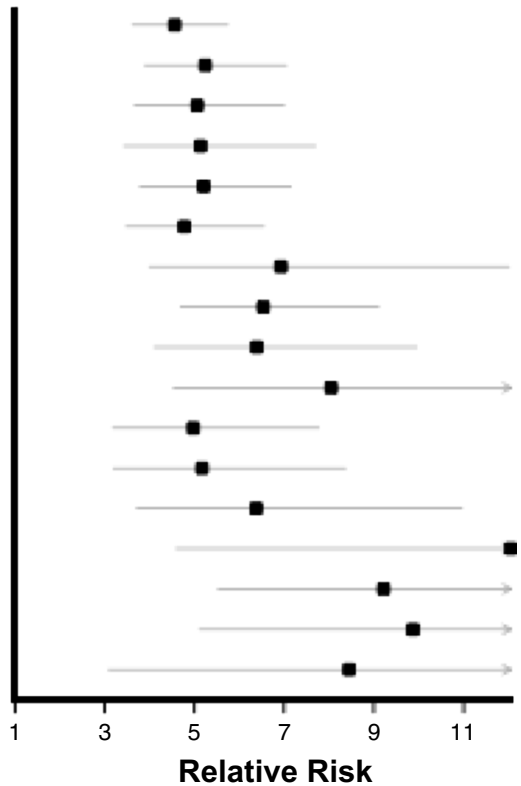

Fig. 4 Predictive power of $24 \mathrm{hpf}$ behavioral data. For chemicals eliciting significant hypoactivity within the B or E time interval in the $24 \mathrm{hpf}$ behavioral data, the relative risk (RR) was estimated for each $5 \mathrm{dpf}$ developmental endpoint. The plots show the estimated RR (solid square) and $95 \%$ confidence intervals. For each plot, the vertical line indicates a $\mathrm{RR}=1$ (i.e., no evidence of association). The 24 hpf behavioral and $5 \mathrm{dpf}$ developmental profiles atop the plots are for the chemical thiram abnormalities-including 18 of 22 chemicals causing notochord defects-and mortality measured at $5 \mathrm{dpf}$. Moreover, many $24 \mathrm{hpf}$ behavioral LELs were more potent than those for gross morphological effects, such as the hypoactive LELs for all three neurotoxicant avermectins tested: abamectin, milbemectin, and emamectin benzoate (Lumaret et al. 2012). Additional value added from this assay is exemplified by responses such as that of thiram (a member of the ethylene bisdithiocarbamate class and the most potent 24 hpf hypoactive chemical), which was not associated with mortality at either $24 \mathrm{hpf}$ or $5 \mathrm{dpf}$, though specific developmental defects (including notochord malformation) were observed at $5 \mathrm{dpf}$ (see Fig. 4).

While confidence bands for the lower-incidence endpoints in Fig. 4 are wider than those for more common 5 dpf endpoints, all endpoint-wise RR for chemicals eliciting hypoactive responses in B or E is statistically significant in the positive direction (i.e., aberrant behavior at $24 \mathrm{hpf}$ is associated with higher risk of developmental abnormalities at $5 \mathrm{dpf}$ ). Furthermore, the positive predictive values (PPV) of hypoactivity results for any $5 \mathrm{dpf}$ endpoint, defined as the fraction of chemicals causing $24 \mathrm{hpf}$ hypoactivity that caused at least one specific developmental abnormality at 5 dpf, were 90,66 , and $71 \%$ for the B, E, and R intervals, respectively.

\section{Discussion}

As an integrative measure of normal development, significant alterations in movement highlight chemicals representing several modes of action. Evaluating the photomotor 
response of chemical-exposed embryos can provide a holistic sensor, unachievable through focused cell-based systems or singular morphological phenotypic assessments. This is of significant value in a screening context employing a comprehensive suite of endpoints, where certain chemical-elicited responses may not manifest in strictly morphological changes observed at any single time point. Even without a specific mode of action linked to each 24 hpf behavioral positive chemical, aberrant behavior in an otherwise stereotypical response should be cause for additional scrutiny and may eventually refine existing AOPs or elucidate entire new pathways.

These results demonstrate that chemicals altering behavioral responses at an early time point $(24 \mathrm{hpf})$ can manifest in later ( $5 \mathrm{dpf})$ developmental abnormalities, indicating that rapid behavioral screening can be a useful diagnostic. Moreover, chemicals associated with severe behavioral abnormalities may lead to developmental effects that are, as yet, unmeasured. In these data, we found 102 chemicals associated with $24 \mathrm{hpf}$ behavioral endpoints yet no specific $5 \mathrm{dpf}$ endpoint associations (Supplemental Table 3). These chemicals, when encountered at a critical, early exposure period, may alter the timing of the coordinated cascade of developmental events that normally lead to healthy adults. Compared to effects detected by manual, visual inspection at $24 \mathrm{hpf}$, we found that the majority of chemicals inducing aberrant behavior were either undetected or not identified until higher concentrations. This demonstrates added value of the $24 \mathrm{hpf}$ behavior assay in a screening paradigm that includes morphological screens as in Truong et al. (2014) or end-stage scoring approaches as in Padilla et al. (2012). In addition to expanding the net for detection of adverse outcomes, combining this early, nondestructive assay with later endpoints should reduce false negatives. Given such a large, diverse chemical set, false negatives within a particular assay can arise for any reason, ranging from technical handling of chemicals preferentially affecting certain endpoints (especially if measured at different time points) to sampling variability within outbred populations. Therefore, the combination of evaluating both early behavioral endpoint and phenotypic responses in the same animals provides the holistic perspective necessary to warrant further study of active chemicals.

While measures have been taken to avoid false positives, the analysis here is tuned toward detection, since the goal was to screen a large set of environmental chemicals in full concentration-response format. This goal diverges from that of compound-similarity clustering approaches such as Kokel et al. (2010), because each chemical in our set of compounds is (or has been) in commerce and the environment. For this reason, they must be characterized to determine whether significant evidence of hazard exists across a broad concentration range. While our analysis took advantage of the magnitude of all data to define expectations and identify outliers, the statistical framework described here can accommodate smaller-scale experiments, because activity thresholds are ratio-based comparisons to local controls, rather than requiring an absolute unit change in spontaneous movement.

The advantages of the statistical framework developed here over more traditional alternatives, such as paired second-to-second tests, include: (1) ability to detect changes in both centrality (i.e., average movement) and distributional shape; (2) robustness in the face of changing, nonstandard distributions in a measurement of a spontaneous phenotype; and (3) consistency, in that explicit perturbations (i.e., light pulses) define more stable responses than periods of only a few seconds within an experimental interval. While the time periods could be subdivided into smaller units of analysis for fine-scale modeling of particular responses, we found that the asynchrony between individual embryo wells on a second-to-second time scale overwhelms treatment-associated variability-especially if more than the samples comprising the interquartile range are analyzed as in the pooled embryo method of (Kokel et al. 2010). However, when considered across a multi-second experimental interval, consistencies arise, and by using all samples to characterize the effects of a given chemical, we capture a more representative sample of the full variability present in the population. Indeed, our analysis shows high reliability amongst separately sourced, blinded replicate sets.

Importantly, with such a noninvasive, automated endpoint, perturbations of AOP-associated molecular targets could be assessed through morpholino knockdown and/or RNA rescue (Andreasen et al. 2006; Franzosa et al. 2013; Miller et al. 2014; Reimers et al. 2006; Tilton et al. 2006; Tilton and Tanguay 2008). The scale of the results presented here, using an experimental design including high numbers of biological well-replicates, permits powerful enrichment analysis to confirm such molecular targets. We have presented integrated analyses spanning endpoints via follow-up within our experiment ( $24 \mathrm{hpf}$ behavior to $5 \mathrm{dpf}$ morphology) and external experiments (biochemical assays from Sipes et al.). This demonstrates that these results can augment the value of systematic data generated by ongoing efforts to realize the promise of modernized, sustainable approaches to environmental health protection as envisaged by the US National Research Council's Toxicity testing in the 21st century: a vision and a strategy (2007). Accordingly, we present these rapid throughput, vertebrate screening results as an important resource for cross-species hazard assessment and encourage the use of these data for additional meta- and cross-platform analyses.

In conclusion, this rapid neurobehavioral assay identified chemicals displaying light-dependent and light-independent effects at an early developmental stage ( $24 \mathrm{hpf})$, 
predicted increased risk of later (5 dpf) developmental hazard, associated behavioral responses with in vitro endpoints targeting specific biological pathways, characterized a broad concentration-response profile for each chemical, and detected chemicals covering several potential modes of action. The experiments described here address statistical uncertainty concerns commonly associated with in vivo methods-such as low sample size and inadequate concentration-response-by engineering an efficient, rapid throughput system where these important design factors can be efficiently optimized. This system represents an efficient experimental platform for conducting neurobehavioral assessment early in vertebrate development that, due to its noninvasive nature, can be recombined with other experimental interventions and/or later outcome measures.

Acknowledgments We would like to acknowledge the EPA for providing the ToxCast chemicals used for these studies. Special thanks go to members of the Tanguay laboratory and the Sinnhuber Aquatic Research Laboratory for assistance with the chemical screening and fish husbandry. This work was supported by NIEHS grants R01 ES19604, R01 ES023788, P42 ES005948, P30 ES025128, RC4 ES019764 P30, P30 ES000210 and Environmental Protection Agency (EPA) STAR Grant \#R835168.

Open Access This article is distributed under the terms of the Creative Commons Attribution 4.0 International License (http://creativecommons.org/licenses/by/4.0/), which permits unrestricted use, distribution, and reproduction in any medium, provided you give appropriate credit to the original author(s) and the source, provide a link to the Creative Commons license, and indicate if changes were made.

\section{References}

Ai Q, Jing Y, Jiang R, Lin L, Dai J, Che Q, Zhou D, Jia M, Wan J, Zhang L (2014) Rotenone, a mitochondrial respiratory complex I inhibitor, ameliorates lipopolysaccharide/D-galactosamineinduced fulminant hepatitis in mice. Int Immunopharmacol 21:200-207

Andreasen EA, Mathew LK, Tanguay RL (2006) Regenerative growth is impacted by TCDD: gene expression analysis reveals extracellular matrix modulation. Toxicol Sci 92:254-269

Bartlett DW, Clough JM, Godwin JR, Hall AA, Hamer M, ParrDobrzanski B (2002) The strobilurin fungicides. Pest Manag Sci 58:649-662

Bretaud S, Lee S, Guo S (2004) Sensitivity of zebrafish to environmental toxins implicated in Parkinson's disease. Neurotoxicol Teratol 26:857-864

Burns CJ, McIntosh LJ, Mink PJ, Jurek AM, Li AA (2013) Pesticide exposure and neurodevelopmental outcomes: review of the epidemiologic and animal studies. J Toxicol Environ Health B $16: 127-283$

Carmichael SN, Bron JE, Taggart JB, Ireland JH, Bekaert M, Burgess ST, Skuce PJ, Nisbet AJ, Gharbi K, Sturm A (2013) Salmon lice (Lepeophtheirus salmonis) showing varying emamectin benzoate susceptibilities differ in neuronal acetylcholine receptor and GABA-gated chloride channel mRNA expression. BMC Genomics 14:408
Collins FS, Gray GM, Bucher JR (2008) Toxicology. Transforming environmental health protection. Science 319:906-907

Cook LW, Paradise CJ, Lom B (2005) The pesticide malathion reduces survival and growth in developing zebrafish. Environ Toxicol Chem SETAC 24:1745-1750

Ducharme NA, Peterson LE, Benfenati E, Reif D, McCollum CW, Gustafsson JA, Bondesson M (2013) Meta-analysis of toxicity and teratogenicity of 133 chemicals from zebrafish developmental toxicity studies. Reprod Toxicol 41:98-108

Ensibi C, Hernandez-Moreno D, Miguez Santiyan MP, Daly Yahya MN, Rodriguez FS, Perez-Lopez M (2014) Effects of carbofuran and deltamethrin on acetylcholinesterase activity in brain and muscle of the common carp. Environ Toxicol 29:386-393

Franzosa JA, Bugel SM, Tal TL, La Du JK, Tilton SC, Waters KM, Tanguay RL (2013) Retinoic acid-dependent regulation of miR-19 expression elicits vertebrate axis defects. FASEB J 27:4866-4876

Haendel MA, Tilton F, Bailey GS, Tanguay RL (2004) Developmental toxicity of the dithiocarbamate pesticide sodium metam in zebrafish. Toxicol Sci 81:390-400

Howe K, Clark MD, Torroja CF, Torrance J, Berthelot C, Muffato M, Collins JE, Humphray S, McLaren K, Matthews L et al (2013) The zebrafish reference genome sequence and its relationship to the human genome. Nature 496:498-503

Judson RS, Houck KA, Kavlock RJ, Knudsen TB, Martin MT, Mortensen HM, Reif DM, Rotroff DM, Shah I, Richard AM et al (2010) In vitro screening of environmental chemicals for targeted testing prioritization: the ToxCast project. Environ Health Perspect 118:485-492

Kimmel CB, Ballard WW, Kimmel SR, Ullmann B, Schilling TF (1995) Stages of embryonic development of the zebrafish. Dev Dyn 203:253-310

Kokel D, Bryan J, Laggner C, White R, Cheung CY, Mateus R, Healey D, Kim S, Werdich AA, Haggarty SJ et al (2010) Rapid behavior-based identification of neuroactive small molecules in the zebrafish. Nat Chem Biol 6:231-237

Kokel D, Dunn TW, Ahrens MB, Alshut R, Cheung CY, Saint-Amant L, Bruni G, Mateus R, van Ham TJ, Shiraki T et al (2013) Identification of nonvisual photomotor response cells in the vertebrate hindbrain. J Neurosci 33:3834-3843

Lee CC, Peters PJ (1976) Neurotoxicity and behavioral effects of thiram in rats. Environ Health Perspect 17:35-43

Levin ED, Chrysanthis E, Yacisin K, Linney E (2003) Chlorpyrifos exposure of developing zebrafish: effects on survival and longterm effects on response latency and spatial discrimination. Neurotoxicol Teratol 25:51-57

Li Y, Cheng CN, Verdun VA, Wingert RA (2014) Zebrafish nephrogenesis is regulated by interactions between retinoic acid, mecom, and Notch signaling. Dev Biol 386:111-122

Lumaret JP, Errouissi F, Floate K, Rombke J, Wardhaugh K (2012) A review on the toxicity and non-target effects of macrocyclic lactones in terrestrial and aquatic environments. Curr Pharm Biotechnol 13:1004-1060

Makris N, Biederman J, Monuteaux MC, Seidman LJ (2009) Towards conceptualizing a neural systems-based anatomy of attentiondeficit/hyperactivity disorder. Dev Neurosci 31:36-49

Mandrell D, Truong L, Jephson C, Sarker MR, Moore A, Lang C, Simonich MT, Tanguay RL (2012) Automated zebrafish chorion removal and single embryo placement: optimizing throughput of zebrafish developmental toxicity screens. J Lab Autom 17:66-74

McGinnis CL, Crivello JF (2011) Elucidating the mechanism of action of tributyltin (TBT) in zebrafish. Aquat Toxicol 103:25-31

Micael J, Reis-Henriques MA, Carvalho AP, Santos MM (2007) Genotoxic effects of binary mixtures of xenoandrogens (tributyltin, triphenyltin) and a xenoestrogen (ethinylestradiol) in a 
partial life-cycle test with Zebrafish (Danio rerio). Environ Int 33:1035-1039

Miller GW, Truong L, Barton CL, Labut EM, Lebold KM, Traber MG, Tanguay RL (2014) The influences of parental diet and vitamin $\mathrm{E}$ intake on the embryonic zebrafish transcriptome. Comp Biochem Physiol D Genomics Proteomics 10:22-29

Navarro A, Bandez MJ, Gomez C, Repetto MG, Boveris A (2010) Effects of rotenone and pyridaben on complex I electron transfer and on mitochondrial nitric oxide synthase functional activity. J Bioenerg Biomembr 42:405-412

NRC (2007) Toxicity testing in the 21st century: a vision and a strategy. The National Academies Press, Washington, D.C

Padilla S, Corum D, Padnos B, Hunter DL, Beam A, Houck KA, Sipes N, Kleinstreuer N, Knudsen T, Dix DJ et al (2012) Zebrafish developmental screening of the ToxCast Phase I chemical library. Reprod Toxicol 33:174-187

Raftery TD, Isales GM, Yozzo KL, Volz DC (2014) High-content screening assay for identification of chemicals impacting spontaneous activity in zebrafish embryos. Environ Sci Technol 48:804-810

R Core Team (2014) R: a language and environment for statistical computing. In: Vienna, Austria: R Foundation for Statistical Computing

Reimers MJ, La Du JK, Periera CB, Giovanini J, Tanguay RL (2006) Ethanol-dependent toxicity in zebrafish is partially attenuated by antioxidants. Neurotoxicol Teratol 28:497-508

Sanes DH, Reh TA, Harris WA (2005) Development of the nervous system. Elsevier, Amsterdam

Selderslaghs IW, Hooyberghs J, Blust R, Witters HE (2013) Assessment of the developmental neurotoxicity of compounds by measuring locomotor activity in zebrafish embryos and larvae. Neurotoxicol Teratol 37:44-56

Shiraishi Y, Murai M, Sakiyama N, Ifuku K, Miyoshi H (2012) Fenpyroximate binds to the interface between PSST and $49 \mathrm{kDa}$ subunits in mitochondrial NADH-ubiquinone oxidoreductase. Biochemistry 51:1953-1963

Sipes NS, Martin MT, Kothiya P, Reif DM, Judson RS, Richard AM, Houck KA, Dix DJ, Kavlock RJ, Knudsen TB (2013) Profiling 976 ToxCast chemicals across 331 enzymatic and receptor signaling assays. Chem Res Toxicol 26:878-895

Swarnkar S, Singh S, Sharma S, Mathur R, Patro IK, Nath C (2011) Rotenone induced neurotoxicity in rat brain areas: a histopathological study. Neurosci Lett 501:123-127
Tilton F, La Du JK, Vue M, Alzarban N, Tanguay RL (2006) Dithiocarbamates have a common toxic effect on zebrafish body axis formation. Toxicol Appl Pharmacol 216:55-68

Tilton F, Tanguay RL (2008) Exposure to sodium metam during zebrafish somitogenesis results in early transcriptional indicators of the ensuing neuronal and muscular dysfunction. Toxicol Sci 106:103-112

Tropepe V, Sive HL (2003) Can zebrafish be used as a model to study the neurodevelopmental causes of autism? Genes Brain Behav 2:268-281

Truong L, Harper SL, Tanguay RL (2011) Evaluation of embryotoxicity using the zebrafish model. Methods Mol Biol 691:271-279

Truong L, Reif DM, St Mary L, Geier MC, Truong HD, Tanguay RL (2014) Multidimensional in vivo hazard assessment using zebrafish. Toxicol Sci 137:212-233

van Schie RM, Bruggemann RJ, Hoogerbrugge PM, te Loo DM (2011) Effect of azole antifungal therapy on vincristine toxicity in childhood acute lymphoblastic leukaemia. J Antimicrob Chemother 66:1853-1856

Waldinger TP, Siegle RJ, Weber W, Voorhees JJ (1984) Dapsoneinduced peripheral neuropathy. Case report and review. Arch Dermatol 120:356-359

Westerfield M (2000) The zebrafish book. A guide for the laboratory use of zebrafish (Danio rerio), 4th edn. University of Oregon, Eugene, OR

Yang D, Lauridsen H, Buels K, Chi LH, La Du J, Bruun DA, Olson JR, Tanguay RL, Lein PJ (2011) Chlorpyrifos-oxon disrupts zebrafish axonal growth and motor behavior. Toxicol Sci 121:146-159

Yasutake A, Marumoto M, Yoshida M (2010) Neurotoxic action of inorganic mercury injected in the intraventricular space of mouse cerebrum. J Toxicol Sci 35:767-771

Ziermans T, Dumontheil I, Roggeman C, Peyrard-Janvid M, Matsson H, Kere J, Klingberg T (2012) Working memory brain activity and capacity link MAOA polymorphism to aggressive behavior during development. Transl Psychiatry 2:e85

Zsombok A, Molnar L, Fischer E (1997) Neurotoxicity of paraquat and triphenyltin in the earthworm, Eisenia fetida Sav. A histoand cytopathological study. Acta Biol Hung 48:485-495 